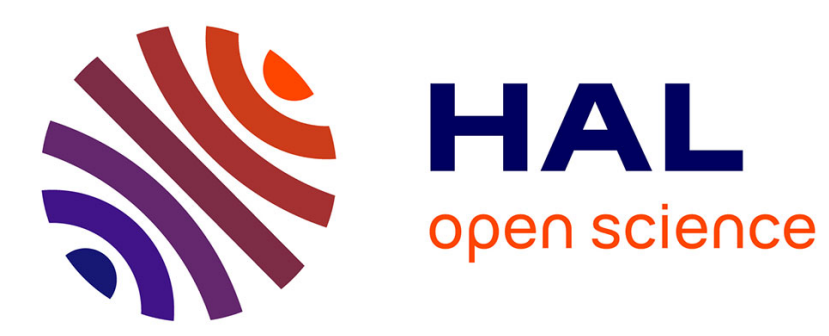

\title{
Implementation of arbitrary real-valued correlation filters for the shadow-casting incoherent correlator
}

\author{
Vincent Laude, Pierre Chavel, Philippe Réfrégier
}

\section{To cite this version:}

Vincent Laude, Pierre Chavel, Philippe Réfrégier. Implementation of arbitrary real-valued correlation filters for the shadow-casting incoherent correlator. Applied optics, 1996, 35 (26), pp.5267-5274. hal00081211

\section{HAL Id: hal-00081211 \\ https://hal.science/hal-00081211}

Submitted on 6 Apr 2012

HAL is a multi-disciplinary open access archive for the deposit and dissemination of scientific research documents, whether they are published or not. The documents may come from teaching and research institutions in France or abroad, or from public or private research centers.
L'archive ouverte pluridisciplinaire HAL, est destinée au dépôt et à la diffusion de documents scientifiques de niveau recherche, publiés ou non, émanant des établissements d'enseignement et de recherche français ou étrangers, des laboratoires publics ou privés. 


\title{
Implementation of arbitrary real-valued correlation filters for the shadow-casting incoherent correlator
}

\author{
Vincent Laude, Pierre Chavel, and Philippe Réfrégier
}

\begin{abstract}
We describe an incoherent correlator, based on the shadow-casting principle, that is able to implement any real-valued linear correlation filter. The correlation filter and the input image are displayed on commercial liquid-crystal television (LCTV) panels. Although it cannot handle high-resolution images, the incoherent correlator is lensless, compact, low cost, and uses a white-light source. A bipolar technique is devised to represent any linear filter, computed from a single reference image or composite, in the correlator. We demonstrate experimentally the efficiency of the design in the case of optimal trade-off (OT) filters and optimal trade-off synthetic discriminant function (OT-SDF) filters. (C) 1996 Optical Society of America

Key words: Shadow-casting correlator, spatial light modulator constrained filters, bipolar filters, optimal trade-off filters, synthetic discriminant function filters.
\end{abstract}

\section{Introduction}

The shadow-casting correlator, ${ }^{1,2}$ as well as the other incoherent correlators, has not benefited as much as coherent correlators from the renewal of interest for optical pattern recognition. Indeed, much work has been devoted to the design and improvement of coherent correlation architectures and to the optical implementation of filtering algorithms for these architectures. In the case of the shadow-casting correlator, it has been known for some time that the resolution is limited by Fresnel diffraction, and pessimistic figures for the number of resolution points that can be used for the filter and the input image were given in Refs. 3 and 4 . However, we show in Section 2 that under given conditions it is possible to determine a significant resolution for processing with a shadow-casting system, provided the target size is

P. Chavel is with the Institute d'Optique Théorique et Appliquée, Groupe de Physique des Images, Centre National de la Recherche Scientifique Unité de Recherche 14, BP 147 F-91403 Orsay cedex, France. When this work was done V. Laude and Ph. Réfrégier were with Thomson-CSF, Laboratoire Central de Recherches, F-91404 Orsay cedex, France. Ph. Réfrégier is now with the Laboratoire Signal et Image, Ecole Nationale Supérieure de Physique de Marseille, F-13397 Marseille cedex 20, France.

Received 6 November 1995; revised manuscript received 12 April 1996.

0003-6935/96/265267-08\$10.00/0

(C) 1996 Optical Society of America small compared with the input image size, in which case the resolution can be traded off between the correlation filter and the input image.

The advantages of incoherent optical information processing with respect to coherent processing are well known. The overall cost is lower, and the system can be made compact, especially if no Fourier lenses are used, as is the case for shadow casting. The sensitivity to mechanical perturbations and optical distortions, such as dust or poor-quality optical components, is much lower. Another practical advantage is that liquid-crystal televisions (LCTV's), often used as spatial light modulators (SLM's) in implementations of optical processing, are optimized for amplitude modulation because they are designed for vision applications rather than for phase modulation, as is often required for efficient coherent processing.

When represented in object space (as opposed to the Fourier space) (i.e., as an impulse response) most linear filters used in optical pattern recognition to date are real-valued filters. ${ }^{5}$ This is true, for example, for the matched, inverse, phase-only, optimal trade-off $(\mathrm{OT})$, synthetic discriminant function (SDF), minimum average correlation energy (MACE), minimum variance synthetic discriminant function (MVSDF), optimal trade-off synthetic discriminant function (OT-SDF), minimum noise and correlation energy (MINACE) filters (see, e.g., Ref. 5 for a description of these correlation filters). Thus it is de- 


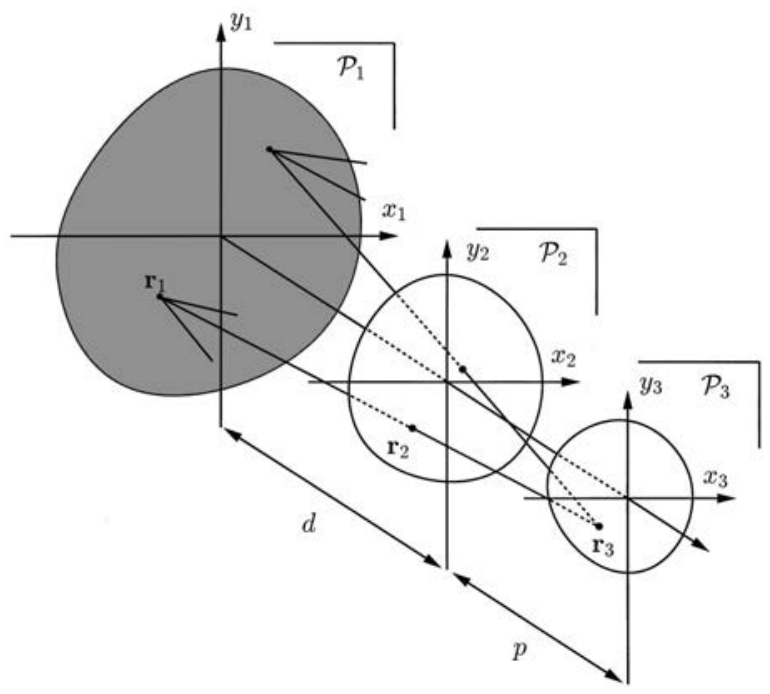

Fig. 1. Shadow-casting principle. In the first plane, $\mathscr{P}_{1}$, is a diffuse source. In the second, $\mathscr{P}_{2}$, is a transparency. In the third, $\mathscr{P}_{3}$, is a screen or a camera.

sirable to be able to represent any given real-valued filter in an optical correlator.

As a result of incoherent illumination, filters that can be represented in a shadow-casting correlator are necessarily positive real-valued images in the object space. To get rid of the same limitation in the related field of pupil synthesis, it was proposed 6 to use a bipolar scheme for the representation of the filter. That is, any real-valued filter $\mathbf{h}$ can be written as the difference of a positive part $\mathbf{h}^{+}$and a negative part $\mathbf{h}^{-}$, both being positive. This enables one to represent any real-valued filter. In practice it is necessary to measure both correlations of the object with the positive and the negative parts of the filter and then to subtract them.

In Section 2 we present a brief analysis of shadow casting in the context of incoherent correlation. In Section 3 we recall some basic properties of linear OT filters. Section 4 describes our bipolar technique for implementing any real-valued linear filter, and Section 5 gives some experimental results for OT filters and OT-SDF filters.

\section{Shadow-Casting Correlator}

Shadow-casting optical systems have been known for quite a long time, at least since 1943. ${ }^{1}$ They allow one to obtain easily the correlation or convolution of two images presented in object space. ${ }^{4}$ As correlators, they were first used in crystallography for the analysis of x-ray diffraction patterns. ${ }^{1,2}$ Apart from correlation, shadow-casting has been applied, for example, to optical implementation of logical operations, ${ }^{7}$ mathematical morphology, ${ }^{8}$ and symbolic substitution. ${ }^{9}$

The simplest shadow-casting setup is depicted in Fig. 1. It involves three planes. In the first plane $\mathscr{P}_{1}$ is a diffuse source that could be, for example, a CRT, ${ }^{10}$ a LED or laser diode array, ${ }^{11}$ or even a transparency or a SLM followed by a diffuser. For the last

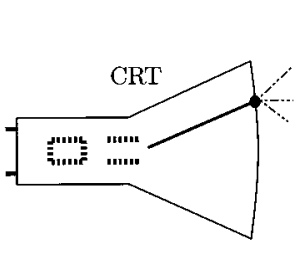

(a)

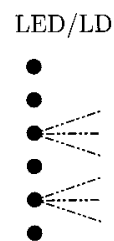

(b)

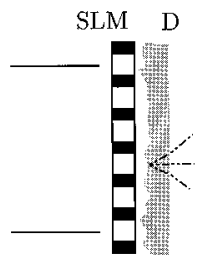

(c)
Fig. 2. Several possibilities for the diffuse source in plane $\mathscr{P}_{1}$. (a) CRT, (b) LED or laser diode array, (c) SLM followed by a diffuser.

an additional incoherent source is needed, for instance, a white-light source. Figure 2 depicts these three possibilities.

In the second plane $\mathscr{P}_{2}$ at a distance $d$ is another SLM or a transparency, and in the third plane $\mathscr{P}_{3}$ at a distance $p$ is a screen or a camera. The distance $d$ is always positive, but the distance $p$ can be positive or negative. In the latter case plane $\mathscr{P}_{3}$ must be observed with an additional optical system. As the first plane is a diffuse source, i.e., spatial incoherence is assumed, the system behaves more or less according to geometrical optics laws, depending on the ratio of characteristic lengths in the setup to the wavelength.

To understand the shadow-casting principle, let us start from the geometrical optics point of view. With the notation of Fig. 1, a ray emitted from point $\mathbf{r}_{1}$, with coordinates $\left(x_{1}, y_{1}\right)$, of the plane of the diffuse source and arriving at point $\mathbf{r}_{3}$ of the detection plane, crosses the second plane at point $\mathbf{r}_{2}$, given by

$$
\frac{\mathbf{r}_{2}}{d^{\prime}}=\frac{\mathbf{r}_{1}}{d}+\frac{\mathbf{r}_{3}}{p}
$$

where the distance $d^{\prime}$ is defined by

$$
\frac{1}{d^{\prime}}=\frac{1}{d}+\frac{1}{p}
$$

Let us consider the elementary surfaces $d \mathbf{r}_{1}$ and $d \mathbf{r}_{3}$ centered at points $\mathbf{r}_{1}$ and $\mathbf{r}_{3}$, respectively. The source and the optical system can be characterized by the elementary flux $\xi\left(\mathbf{r}_{1}, \mathbf{r}_{3}\right) \mathrm{d} \mathbf{r}_{1} \mathrm{~d} \mathbf{r}_{3}$ that is emitted in $\mathrm{d} \mathbf{r}_{1}$ and received in d $\mathbf{r}_{3}$. Two transparencies $M_{1}$ and $M_{2}$ lie, respectively, in planes $\mathscr{P}_{1}$ and $\mathscr{P}_{2}$. The illumination at point $\mathbf{r}_{3}$ is then given by the contribution of all source elements $d \mathbf{r}_{1}$ :

$$
E\left(\mathbf{r}_{3}\right)=\int_{\mathscr{P}_{1}} \mathrm{~d} \mathbf{r}_{1} \xi\left(\mathbf{r}_{1}, \mathbf{r}_{3}\right) M_{1}\left(\mathbf{r}_{1}\right) M_{2}\left[d^{\prime}\left(\frac{\mathbf{r}_{1}}{d}+\frac{\mathbf{r}_{3}}{p}\right)\right] .
$$

This expression shows that the operation achieved by shadow casting is, in general and within the limits of the geometrical approximation, a bilinear transformation with a positive kernel $\xi$ of two positive functions $M_{1}$ and $M_{2}$. The kernel $\xi$ includes everything about the photometry properties of the system. To yield a correlation, kernel $\xi$ should be constant. For instance, in the case of the SLM-diffuser option, this means that the diffuser should be Lambertian. 
Let us define the scaling factor $G$ by

$$
G=\frac{d}{\left|d^{\prime}\right|}=\left|\frac{d+p}{p}\right| \text {. }
$$

If we assume that transparency $M_{2}$ is identical to $M_{1}$ but scaled by a factor $G$, it is easily seen with expression (3) that the autocorrelation of $M_{1}$ is obtained.

Green $^{3}$ studied the maximum resolution achievable with a shadow-casting system. His analysis is based on the number of resolvable points in the presence of diffraction. ${ }^{12}$

Green starts by considering circular resolution cells in both planes $\mathscr{P}_{1}$ and $\mathscr{P}_{2}$, with respective radii $\rho_{1}$ and $\rho_{2}$. Using the Rayleigh criterion, he then shows that

$$
\rho_{1} \rho_{2}=\alpha \lambda d,
$$

where $\lambda$ is the illumination wavelength and $\alpha$ is a particular constant, usually chosen equal to 0.61 , corresponding to the Airy diffraction pattern halfwidth. ${ }^{13}$ Let us now consider SLM's with rectangular pixels in planes $\mathscr{P}_{1}$ and $\mathscr{P}_{2}$, with their dimensions being fixed through the Rayleigh criterion. The pixels surfaces are $S_{1}$ and $S_{2}$, respectively. We assume that we can write

$$
S_{1} S_{2}=4 \alpha^{2} \lambda^{2} d^{2}
$$

although pixels are not circular but rectangular; i.e. the value of $\alpha$ is slightly different from 0.61 . From expression (6) it is now possible to evaluate the product of the numbers of resolution cells in planes $\mathscr{P}_{1}$ and $\mathscr{P}_{2}$. These numbers are, respectively,

$$
N_{1}=\mathscr{A}_{1} / S_{1}, \quad N_{2}=\mathscr{A}_{2} / S_{2},
$$

where $\mathscr{A}_{1}$ and $\mathscr{A}_{2}$ are the total surfaces used in planes $\mathscr{P}_{1}$ and $\mathscr{P}_{2}$. We then obtain

$$
N_{1} N_{2}=\frac{\mathscr{A}_{1} \mathscr{A}_{2}}{4 \alpha^{2} \lambda^{2} d^{2}}
$$

Assume that in planes $\mathscr{P}_{1}$ and $\mathscr{P}_{2}$ the SLM aperture is $2 \mathrm{~cm} \times 2 \mathrm{~cm}$, that distance $d$ is $5 \mathrm{~cm}$, and that the wavelength is $0.5 \mu \mathrm{m}$. If we assume, in addition, that $N_{1}=N_{2}$, then

$$
N_{1}=N_{2} \approx 115 \times 115 .
$$

For instance, it is possible to use images of approximately $115 \times 115$ pixels in both planes $\mathscr{P}_{1}$ and $\mathscr{P}_{2}$. For these numbers the diameter of each pixel is $\sim 175$ $\mu \mathrm{m}$.

It is useful to note that the number of resolution cells given by Eq. (8) does not depend on the distance from the second plane to the detector, or, equivalently, on the scaling factor $G$. The latter can be used to share in a different way the total available resolution between planes $\mathscr{P}_{1}$ and $\mathscr{P}_{2}$. For example, for $G=4$, it is possible to use $57 \times 57$ pixels for the first image and $227 \times 227$ pixels for the second. It is important to be aware that the resolution of shadow- casting systems given by expression (8) is only approximate and is particularized for circular pixels.

\section{Optimal Trade-Off Filters}

The generic problem of pattern recognition that we now consider is the determination of the location of a target in an input image. We consider square images with a total of $N$ pixels and use lexicographic scanning. We use the following notation:

$x$ is a (complex) scalar.

$x^{*}$ is the complex conjugate of scalar $x$.

$|x|$ is the modulus of scalar $x$.

$\mathbf{x}$ is a vector, with the first coordinate $x_{0}$ and the last coordinate $x_{N-1}$.

$\mathbf{x}^{\dagger}$ is a complex-conjugate transposed vector $\mathbf{x}$.

$X \quad$ is an $N \times N$ matrix.

Notation with a carat $\left(^{\wedge}\right)$ indicates that expressions are taken in the Fourier domain. The correlation operation of images $\mathbf{h}$ and $\mathbf{x}$ is written $[\mathbf{h} \circledast \mathbf{x}]$ and is classically defined by

$$
[\mathbf{h} \circledast \mathbf{x}]_{k}=c_{k} \triangleq \frac{1}{N} \sum_{m=1}^{N} h_{m}{ }^{*} x_{m+k} .
$$

By application of the convolution theorem, we also have

$$
\hat{c}_{n}=\hat{h}_{n} * \hat{x}_{n} .
$$

The presence of noise in the input image leads to fluctuations in the correlation image. When the noise is assumed to be stationary, the variance of these fluctuations is a constant, denoted mean square error $^{14,15}$ (MSE):

$$
\operatorname{MSE}(\mathbf{h}) \triangleq \hat{\mathbf{h}}^{\dagger} \hat{S} \hat{\mathbf{h}}
$$

where $\hat{S}$ is the assumed spectral density of the noise, a diagonal matrix of size $N \times N$. The criterion usually used to characterize the noise robustness of a correlation filter is the signal-to-noise ratio ${ }^{14,15}$ (SNR):

$$
\operatorname{SNR}(\mathbf{h}) \triangleq \frac{\left|\hat{\mathbf{h}}^{\dagger} \hat{\mathbf{r}}\right|^{2}}{\hat{\mathbf{h}}^{\dagger} \hat{S} \hat{\mathbf{h}}},
$$

where $\mathbf{r}$ is the reference image. The filter that optimizes this criterion is the classical matched filter ${ }^{14}$ :

$$
\hat{h}_{n}=\frac{\hat{r}_{n}}{\hat{S}_{n n}} .
$$

Despite its excellent noise robustness, the matched filter performs poorly in terms of discrimination. In the presence of background, it was shown that the sharpness of the correlation peak, measured by the peak-to-correlation energy (PCE), can be a useful criterion for evaluating the discrimination capabilities 


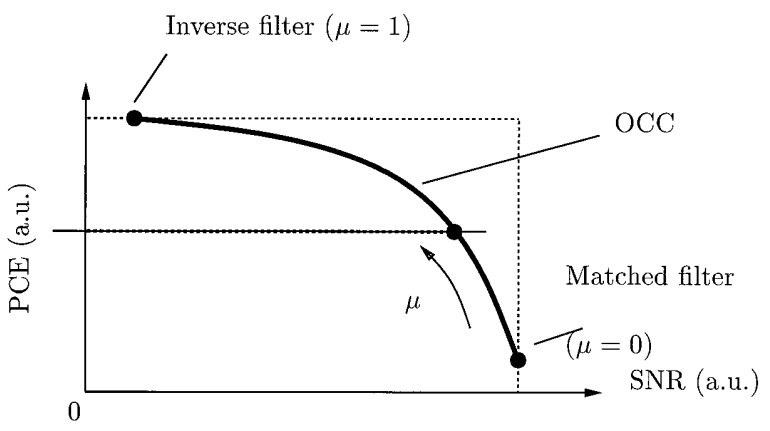

Fig. 3. Principle of OT filters.

of a filter:

$$
\operatorname{PCE}(\mathbf{h}) \triangleq \frac{\left|\hat{\mathbf{h}}^{\dagger} \hat{\mathbf{r}}\right|^{2}}{\hat{\mathbf{h}}^{\dagger} \hat{D} \hat{\mathbf{h}}},
$$

where $\hat{D}$ is the power spectral density of the reference image, a diagonal matrix with elements $\hat{D}_{n n}=\left|\hat{r}_{n}\right|^{2}$. The inverse filter optimizes this last criterion ${ }^{14}$ :

$$
\hat{h}_{n}=\frac{\hat{r}_{n}}{\left|\hat{r}_{n}\right|^{2}} .
$$

The inverse filter is highly unstable ${ }^{16}$ in the presence of noise. Instead of the PCE criterion, we could have used other discrimination criteria as given, for example, in Refs. 17 and 18.

We briefly recall here the principle of OT filters. ${ }^{19,20}$ Let us first jointly consider the SNR and the PCE criteria. Instead of simply optimizing these criteria separately, let us impose the value $\operatorname{PCE}(\mathbf{h})$. Then among all filters that lead to that particular value of criterion $\mathrm{PCE}$, the most interesting filter is definitely the filter that optimizes the criterion SNR. This situation is shown in Fig. 3. In the SNR-PCE plane, for a given value of $\mathrm{PCE}(\mathbf{h})$, all corresponding filters are represented by one point in a horizontal line. Among those filters, the most interesting is the one with the largest value $\operatorname{SNR}(\mathbf{h})$. Repeating this procedure for all possible values of $\operatorname{PCE}(\mathbf{h})$, we obtain a concave curve that represents the OT between criteria SNR and PCE. ${ }^{19}$ This curve is called the optimal characteristics curve (OCC).

The expression of OT filters for the SNR and PCE criteria can be shown to be ${ }^{19}$

$$
\hat{h}_{n}^{\text {от }}=\frac{\hat{r}_{n}}{(1-\mu) \hat{S}_{n n}+\mu \hat{D}_{n n}} .
$$

These filters are denoted in the following $\mathbf{h}^{\mathrm{OT}}$ for OT filters. When parameter $\mu$ varies between 0 and 1 , the OCC depicted in Fig. 3 is generated. For $\mu=0$, the matched filter is obtained, and for $\mu=1$ the inverse filter is obtained. OT filters are real-valued filters in object space, i.e., after the inverse Fourier transform of expression (15) is taken. It is interesting to note that if one starts with a positive reference image then only the matched filter remains positive, whereas for values of $\mu$ different from 0 the OT filter has positive or negative pixel values.

\section{Bipolar Implementation of Linear Filters}

In a recent article ${ }^{21}$ we extended the principle of OT filters and added the criterion of optical efficiency to the SNR and the PCE to coherent correlators, whose filter is displayed in a Fourier plane on a SLM. In that paper we gave an algorithm for obtaining the OT filters for any SLM, characterized by its coding domain. Although the same theoretical approach can be used for shadow-casting correlation filters, this is not necessary if optical efficiency is not taken into account and the following bipolar procedure is used. Optical efficiency is a less important criterion for incoherent than for coherent correlation because lowcost, powerful incoherent sources are widespread.

Because of the incoherence of the source, only images with pixel values in $[0,1]$ can be displayed. Twisted nematic liquid-crystal SLMs are good candidates because they offer video-rate operation and a sufficient contrast. ${ }^{22}$ The bipolar filtering technique described below allows one to overcome the limitation of positiveness for the filter and proves efficient because it can optically implement any real-valued linear filter.

As the correlation image measured with a shadowcasting correlation system is linear with the input image and the filter, it is possible to represent a filter with positive and negative pixel values with a bipolar scheme ${ }^{4}$ :

$$
\mathbf{h}=\mathbf{h}^{+}-\mathbf{h}^{-}, \quad \text { with } \mathbf{h}^{+}, \mathbf{h}^{-} \in[0,1]^{N} .
$$

The resulting bipolar filter $\mathbf{h}$ is thus an element of $[-1,1]^{\mathrm{N}}$, corresponding to the virtual coding domain $[-1,1]$. The principle of bipolar filtering is to record both correlations $\mathbf{c}^{+}=\mathbf{h}^{+} \circledast \mathbf{x}$ and $\mathbf{c}^{-}=\mathbf{h}^{-} \circledast \mathbf{x}$, then to take their squared difference, leading to

$$
\begin{aligned}
|\mathbf{c}|^{2} & =\left|\mathbf{c}^{+}-\mathbf{c}^{-}\right|^{2} \\
& =\left|\mathbf{h}^{+} \circledast \mathbf{x}-\mathbf{h}^{-} \circledast \mathbf{x}\right|^{2} \\
& =|\mathbf{h} \circledast \mathbf{x}|^{2} .
\end{aligned}
$$

The square operation is required from optimal location estimation theory. ${ }^{23}$ The drawback of the bipolar method is that it is necessary to measure two correlation images rather than one. But the filtering capabilities of the filter are dramatically improved.

Note that there exists an infinite number of possible bipolar decompositions for a given filter. Indeed, it is always possible to add an image $\mathbf{f}$ such that

$$
\begin{aligned}
\mathbf{h}=\left[\mathbf{h}^{+}+\mathbf{f}\right]-\left[\mathbf{h}^{-}+\mathbf{f}\right], \\
\text { with }\left[\mathbf{h}^{+}+\mathbf{f}\right],\left[\mathbf{h}^{-}+\mathbf{f}\right] \in[0,1]^{N} .
\end{aligned}
$$

Any real-valued linear filter can be represented with a bipolar decomposition simply by adjustment of its dynamics to the range $[-1,1]$. In the case of an OT filter as given by Eq. (15), the bipolar filter is 


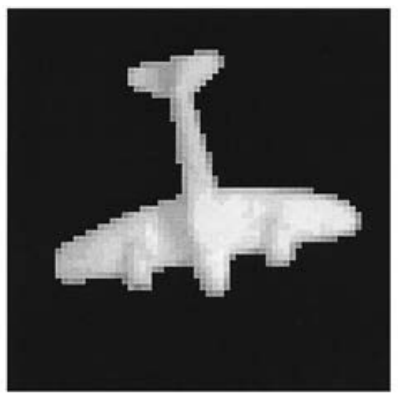

(a)

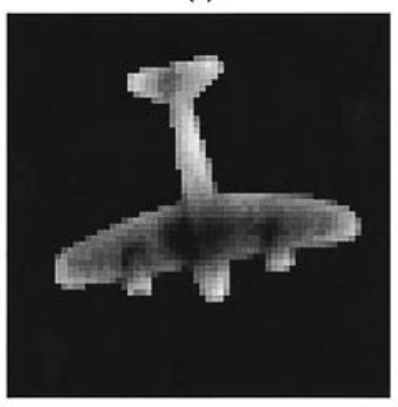

(c)

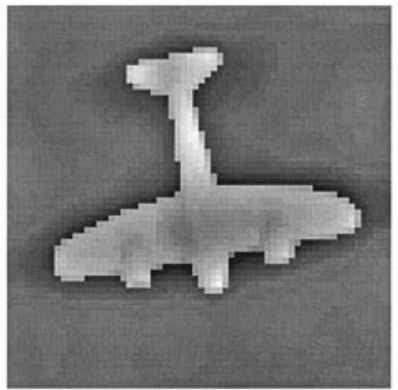

(b)

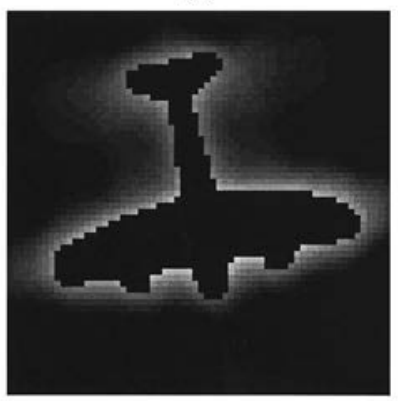

(d)
Fig. 4. Example of bipolar filter design. (a) Reference image. (b) OT filter designed from reference image. Since this filter has positive and negative values, the zero value is offset to grey level 127. (c) Positive part of bipolar filter. (d) Negative part of bipolar filter.

simply

$$
\mathbf{h}=\operatorname{DFT}^{-1}\left(\gamma \hat{\mathbf{h}}^{\mathrm{OT}}\right) .
$$

In this expression $\mathrm{DFT}^{-1}$ denotes the discrete inverse Fourier transform, $\hat{\mathbf{h}}^{\mathrm{OT}}$ is given by Eq. (15), and the scaling parameter $\gamma$ is adjusted such that values of $\mathbf{h}$ are in $[-1,1]$.

Let us define the filters $\mathbf{h}^{+}$and $\mathbf{h}^{-}$by

$$
\begin{aligned}
& h_{m}{ }^{+}=\max \left(0, h_{m}\right), \\
& h_{m}{ }^{-}=\max \left(0,-h_{m}\right) .
\end{aligned}
$$

These definitions are the classical definitions of the positive and negative parts of a function. ${ }^{4}$ It is the decomposition that yields the smallest possible values of $\mathbf{h}^{+}$and $\mathbf{h}^{-}$for every pixel $m$. It is still possible to add a positive image $\mathbf{f}$ to $\mathbf{h}^{+}$and $\mathbf{h}^{-}$. However, it is clear that this will not add any useful information in the correlation plane and will only result in a misuse of the camera dynamics range.

Figure 4 shows an example of the bipolar decomposition of a filter. The OT filter of Fig. 4(b) is designed from the reference image of Fig. 4(a), assuming a white-noise model [i.e., the spectral density $\hat{S}$ is constant in Eq. (15)].

\section{Experiments}

Figure 5 shows the shadow-casting correlator that we designed. All optical elements are low in cost and standard. The white-light source beam is colli-



Fig. 5. Shadow-casting correlator. Filter $\mathbf{h}$ and input image $\mathbf{x}$ are displayed on Epson LCTV SLM's. A, analyzer; P, polarizer; D, diffuser; RGB, red, green, and blue inputs to the Epson video projector.

mated with a simple lens that achieves a sufficient illumination uniformity on the first SLM. The diffuser is a piece of ground glass of which the rough side is in near contact with the first SLM. The measured half-width of the diffusion angle is $\sim 10^{\circ}$. The detector is a CCD array. The SLM's are Epson Model VPJ-2000 LCTV's driven by the video projector original electronics. The measured contrast with the white-light source is $\sim 40$. A color frame grabber is used to write images to the video projector. Two color channels (e.g., red and green) are used, respectively, for the input image and the filter.

The geometrical dimensions chosen for the setup are $d=5.5 \mathrm{~cm}$ and $G=4$, implying that $\mathrm{P}=1.83 \mathrm{~cm}$. These values allow us to use a filter of $64 \times 64$ pixels and an input image of $256 \times 256$ pixels, following the resolution analysis of Section 2 . The positive and negative parts of the bipolar filters are thus zoomed by a factor of 4 before display. The active part of the setup, from the first SLM to the CCD array plane, is smaller than $7.5 \mathrm{~cm}$. The total size, including the source and the CCD camera, is less than $20 \mathrm{~cm}$ and could be easily optimized further.

Figures 6 and 7 illustrate the performance of the bipolar technique. Both input images shown in Figs. 6(a) and 7(a) present an important structured background with a high mean value. The reference airplane shown in Fig. 4 was extracted from the first input image. In the second input image, the airplane appears smaller and is viewed from a slightly different angle. The bipolar decomposition of the OT filter of Fig. 4 is used in the incoherent correlator. For both examples the correlation with the matched filter would fail in the recognition task. The use of an OT filter yields much better discrimination capabilities. The bipolar OT filter, like the classical OT 


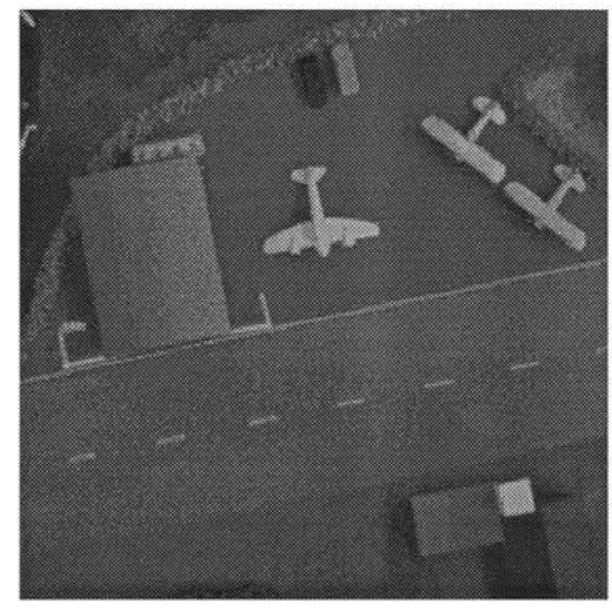

(a)

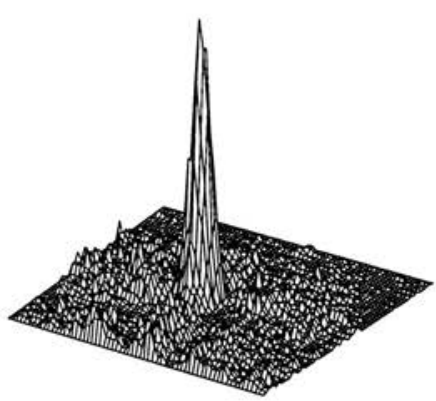

(b)

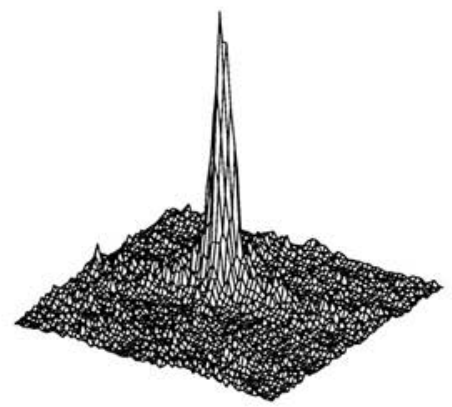

(c)

Fig. 6. Example of a bipolar filtering result. (a) Input image, from which the reference image of Fig. 4(a) was extracted; (b) threedimensional view of the measured correlation of the bipolar filter of Fig. 4 and the input image; (c) three-dimensional view of the simulated correlation of the bipolar filter of Fig. 4 and the input image.

filter, retains a large part of the good noise robustness of the matched filter while improving dramatically over its discrimination capabilities. Here, robustness must be understood as robustness to a modeling defect (distortion of the reference image in the second case).

Figure 8 presents another application of the bipolar OT filter in the context of airplane recognition. The input image shows an airplane viewed in a cloudy background. Note that, as above, the OT bipolar filter is designed with a white-noise model, which is quite different from the actual cloud spectral density.

We now turn to the implementation of SDF filters. The mathematical background for these filters can be found, e.g., in Refs. 5, 19, and 24. SDF filters are usually devised for classification applications. The filter is computed from a learning base that com- prises a certain number of examples that are thought to be typical of an expected distortion. Constraints are imposed on the filter: the center of the correlation function with each member of the learning base must be equal to a specified value. These constraints are generally too small in number for the SDF filter to be precisely determined. Indeed, if $N$ is the number of pixels of example images and $p$ is the number of constraints, the number of degrees of freedom is roughly $N-p$. This is fortunate, because this freedom can be used for optimizing a criterion (e.g., MACE and MV-SDF filters) or for trading-off several criteria (e.g., OT-SDF and MINACE filters). All SDF-type filters mentioned above are real-valued filters in object space and can thus be implemented with our bipolar method.

Let us consider the example of Fig. 9. The learn-

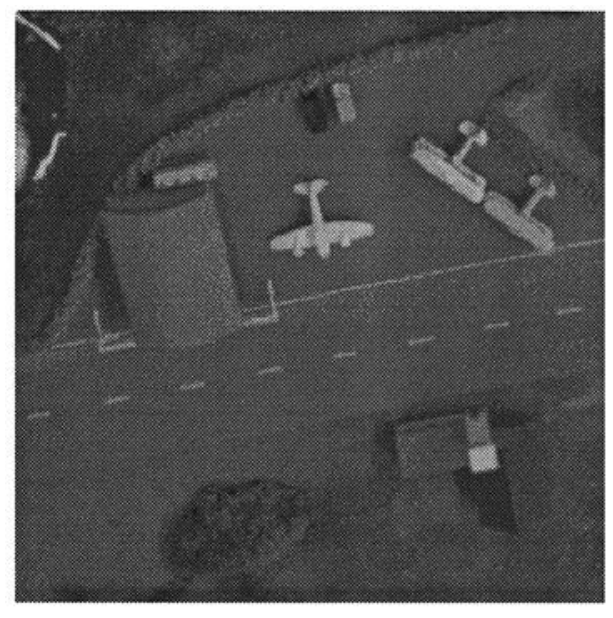

(a)

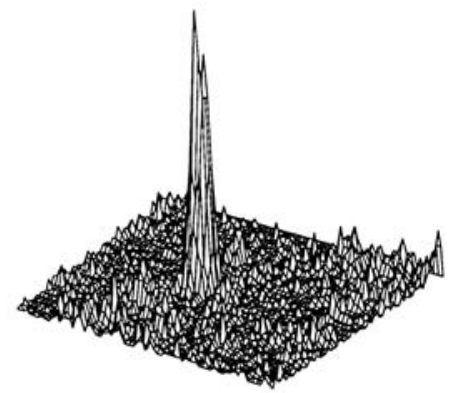

(b)

Fig. 7. Example of a bipolar filtering result. (a) Input image, slightly different from that of Fig. 6(a); (b) three-dimensional view of the measured correlation of the bipolar filter of Fig. 4 and the input image. 


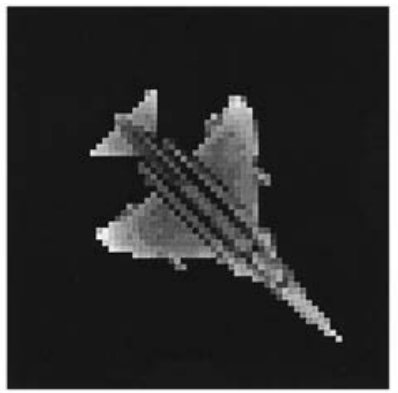

(a)

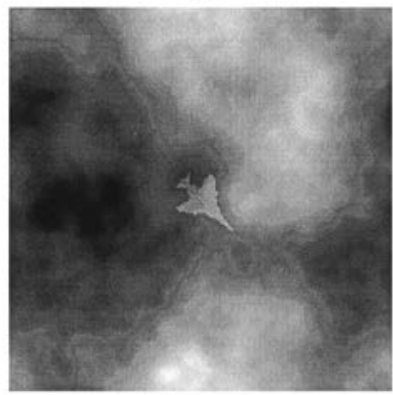

(c)

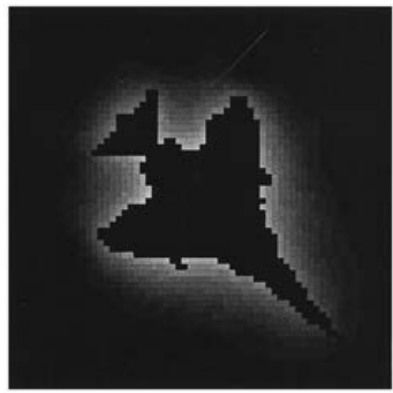

(b)

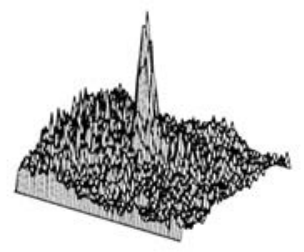

(d)
Fig. 8. Example of a bipolar filtering result. (a) Positive part of bipolar filter; (b) negative part of bipolar filter; (c) input image from which the reference image was extracted; (d) three-dimensional view of the measured correlation of the bipolar filter and the input image.

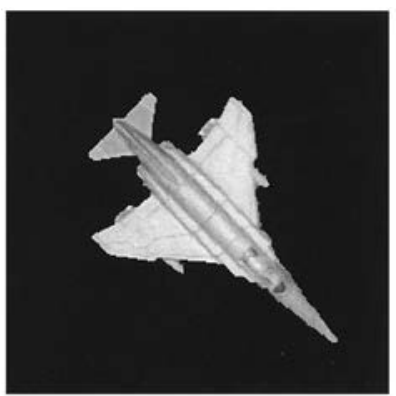

(a)

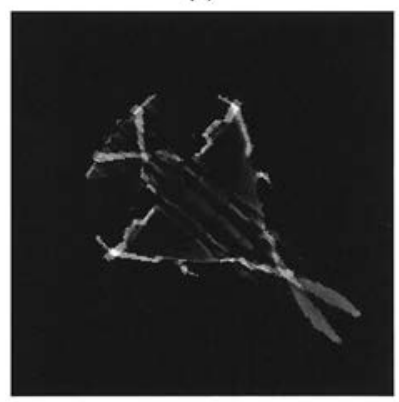

(c)

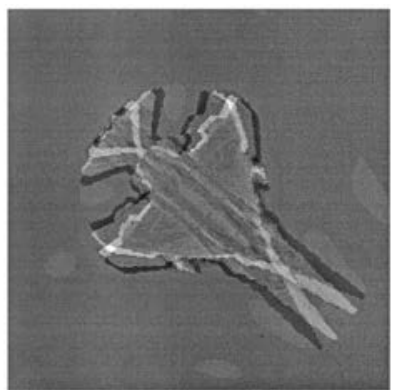

(b)

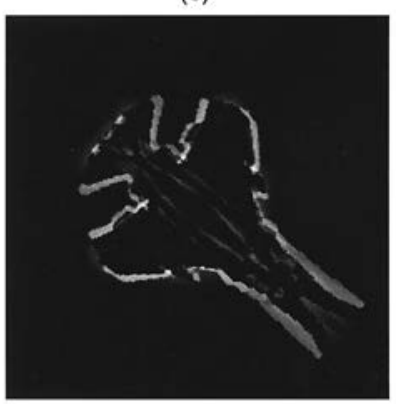

(d)
Fig. 9. Example of a OT-SDF filter designed for attitude recognition (see text) and its bipolar decomposition. (a) A pattern of the learning base; (b) OT-SDF filter designed from learning base; (c) positive part of bipolar OT-SDF filter; (d) negative part of bipolar OT-SDF filter. (a)

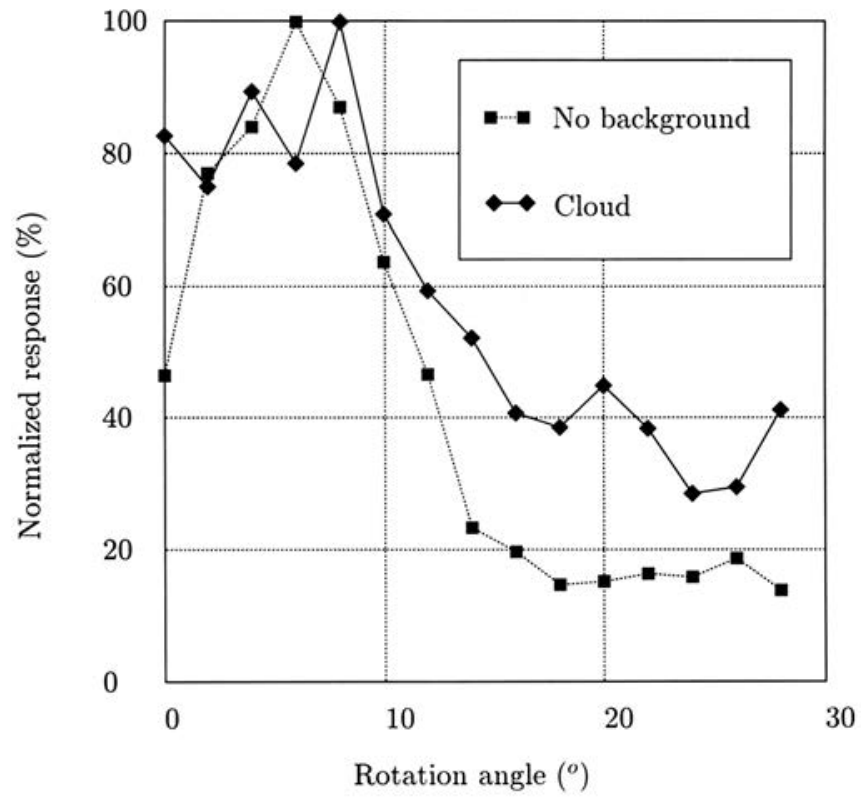

(c)

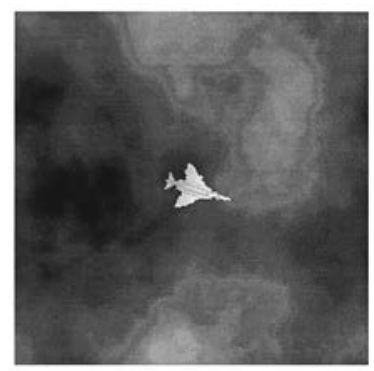

(b)

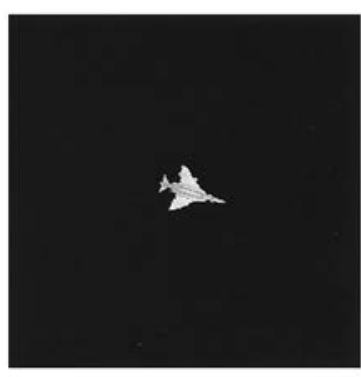

Fig. 10. Experimental response of the bipolar OT-SDF filter of Fig. 9 when the airplane is viewed on a black or a cloudy background. (a) Example of a test image without background and the airplane rotated at $28^{\circ}$; (b) example of a test image with a cloudy background and the airplane rotated at $28^{\circ}$; (c) normalized response of OT-SDF filter.

ing base comprises five images obtained from the image shown in Fig. 9(a) by in-plane rotations of $-5^{\circ}$, $0^{\circ}, 5^{\circ}, 10^{\circ}$, and $15^{\circ}$. These five images are distributed in two classes: the centers of the correlation function with images $\left(0^{\circ}, 5^{\circ}, 10^{\circ}\right)$ are constrained to value 1 , while the centers of the correlation function with images $\left(-5^{\circ}, 15^{\circ}\right)$ are constrained to value 0 . A OT-SDF filter thus obtained is shown in Fig. 9(b), and its positive and negative parts are shown, respectively, in Figs. 9(c) and 9(d). This filter is intended for detecting the presence of an airplane in the angular sector $\left[0^{\circ}, 10^{\circ}\right] .{ }^{25}$

We show in Fig. 10 the experimental response of the bipolar OT-SDF filter of Fig. 9 when the airplane is viewed on a black or a cloudy background. Figures 10(a) and 10(b) are examples of test images in these two cases, respectively, for an in-plane rotation angle of $28^{\circ}$. The response is defined as the maximum value of the squared correlation function, regardless of the location of the maximum. It is clear 
from these results that a correct recognition of the $\left[0^{\circ}\right.$, $10^{\circ}$ ] angular sector is obtained.

We did various other experiments, varying the characteristics of the real-valued linear filter to be implemented with the bipolar technique. We found that the influence of acquisition noise from the CCD camera was generally small, although it might have been expected that substracting the correlations with the positive and negative parts of the filter would have amplified it. More significantly, a problem can arise with the acquisition dynamic range. Indeed, the positive (negative) correlation usually has a large mean value over the input image, over which the positive (negative) target peak appears at the location of the target. If care is not taken, the height of the target peak can become as small as a quantization level of the camera, in which case the target peak is too small to be detected. This thresholding effect can of course be avoided by the use of a larger acquisition dynamic range, provided the acquisition noise remains small enough.

\section{Conclusion}

We have designed an incoherent correlator based on the shadow-casting principle that includes a bipolar representation of the correlation filters. This correlator is very simple and low in cost. The bipolar technique allows us to represent any real-valued linear filter in the correlator. We demonstrated experimentally its efficiency in the case of OT and OT-SDF filters.

Compared with coherent correlators, our shadowcasting incoherent correlator is better in terms of cost, size, and overall robustness, but it suffers from a necessarily limited resolution. For applications in which the target is relatively small compared with the input image and in which it is preferable to emphasize filtering robustness rather than high discrimination capabilities, it seems to be a good candidate. However, when high-resolution or highdiscrimination capabilities are needed, in general coherent correlators would be more appropriate.

This research was partially supported by the Direction de la Recherche et de la Technologie under contract 92/352. The authors thank D. Broussoux, G. Delaforest, and S. Formont for helpful discussions.

\section{References}

1. M. M. Robertson, "Interpretation of Patterson diagrams," Nature 152, 411-412 (1943).

2. L. Bragg, "Lightning calculations with light," Nature 154, 69-72 (1944).

3. E. L. Green, "Diffraction in lensless correlation," Appl. Opt. 7, 1237-1239 (1968).

4. G. L. Rogers, Noncoherent Optical Processing (Wiley, New York, 1977).
5. B. V. K. Vijaya Kumar, C. Hendrix, and D. W. Carlson, "Tradeoffs in the design of correlation filters," in Optical Pattern Recognition, J. L. Horner and B. Javidi, eds. (Society of Photo-Optical Instrument Engineers Optical Engineering, Bellingham, Wash., 1992), pp. 191-215.

6. J. N. Mait, "Pupil-function design for bipolar incoherent spatial filtering,” J. Opt. Soc. Am. A 3, 1826-1832 (1986)

7. Y. Ichioka and J. Tanida, "Optical parallel logic gates using a shadow-casting system for optical digital computing," Proc. IEEE 72, 787-801 (1984).

8. Y. Li, A. Kostrzewski, D. H. Kim, and G. Eichmann, "Compact parallel real-time programmable optical morphological image processor," Opt. Lett. 14, 981-983 (1989).

9. A. Louri, "Efficient optical implementation method for symbolic substitution logic based on shadow casting," Appl. Opt. 14, 3264-3267 (1989).

10. P. L. Jackson, "Correlation function spatial filtering with incoherent light," Appl. Opt. 6, 1272-1973 (1967).

11. D. Raj, D. W. Prather, R. A. Athale, and J. N. Mait, "Performance analysis of optical shadow-casting correlators," Appl. Opt. 32, 3108-3112 (1993).

12. M. Born and E. Wolf, Principles of Optics (Pergamon, New York, 1980).

13. M. Gedziorowski and T. Szoplik, "Resolution of a lensless shadow casting correlator with partially coherent illumination," Opt. Commun. 106, 167-172 (1994).

14. B. V. K. Vijaya Kumar and L. Hassebrook, "Performance measures for correlation filters," Appl. Opt. 29, 2997-3006 (1990).

15. J. L. Horner, "Metrics for assessing pattern-recognition performance," Appl. Opt. 31, 165-166 (1992).

16. Ph. Réfrégier, "Application of the stabilizing functional approach to pattern recognition filters," J. Opt. Soc. Am. A 11, 1243-1251 (1994).

17. Ph. Réfrégier, V. Laude, and B. Javidi, "Basic properties of nonlinear global filtering techniques and optimal discriminant solutions," Appl. Opt. 34, 3915-3923 (1995).

18. L. P. Yaroslavsky, "Optical correlators with $(-k)$ th-law nonlinearity: optimal and suboptimal solutions," Appl. Opt. 34, 3924-3932 (1995)

19. Ph. Réfrégier, "Filter design for optical pattern recognition: multicriteria optimization approach," Opt. Lett. 15, 854-856 (1990).

20. J. Figue and $\mathrm{Ph}$. Réfrégier, "On the optimality of trade-off filters,” Appl. Opt. 32, 1933-1935 (1993).

21. V. Laude and Ph. Réfrégier, "Multicriteria characterization of coding domains with optimal Fourier spatial light modulator filters," Appl. Opt. 33, 4465-4471 (1994).

22. V. Laude, S. Mazé, P. Chavel, and Ph. Réfrégier, "Amplitude and phase coding measurements of a liquid crystal television," Opt. Commun. 103, 33-38 (1993).

23. L. P. Yaroslavsky, "The theory of optimal methods for localization of objects in images," in Progress in Optics XXXII, (North Holland, Amsterdam, 1993), pp. 145-201.

24. D. Casasent, "Unified synthetic discriminant function computation formulation," Appl. Opt. 23, 1620-1627 (1984).

25. J. Figue and Ph. Réfrégier, "Angle determination of airplanes by multicorrelation techniques with optimal trade-off synthetic discriminant filters," Opt. Eng. 33, 1821-1828 (1994). 